\title{
APOSYNDETIC CONTINUA AS BUNDLE SPACES ${ }^{(1)}$
}

BY

JAMES T. ROGERS, JR.

\begin{abstract}
Let $\delta$ be the $P$-adic solenoid bundle, and let $\eta: X \rightarrow S^{1}$ be a map of the continuum $X$ onto $S^{1}$. The bundle space $B$ of the induced bundle $\eta^{-1} S$ is investigated. Sufficient conditions are obtained for $B$ to be connected, to be aposyndetic, and to be homogeneous. Uncountably many aposyndetic, homogeneous, one-dimensional, nonlocally connected continua are constructed. Other classes of continua are placed into this framework.
\end{abstract}

1. Let $\mathcal{S}=\left(S, f, S^{1}, G\right)$ be the $P$-adic solenoid bundle, where $S$ is the $P$-adic solenoid, $G$ is the $P$-adic integers, and $f: S \rightarrow S^{1}$ is the projection of $S$ onto $S^{1}$. If $X$ is a continuum and $\eta: X \rightarrow S^{1}$ is a map, then the bundle space $B$ of the induced bundle $\eta^{-1} \delta$ is a nonlocally connected compactum of the same dimension as $X$.

When $B$ is a continuum, it possesses interesting properties. It is neither arcwiseconnected nor movable, and its first Čech cohomology group $H^{1}(X ; Z)$ contains an element that admits infinite division. Under quite reasonable conditions on $X$, the continuum $B$ is aposyndetic (thus we have another class of aposyndetic and nonlocally connected continua occurring "in nature" [5]), and if $X$ is strongly locally homogeneous, then $B$ is homogeneous. The importance of aposyndesis in the study of homogeneous continua is explained in [8].

This paper develops tools to recognize when $B$ is a continuum and what its topological properties are. We view the process as a machine with input $X$ and $\eta$ and output $B$ and $h$.

For example, if $X$ is the Menger universal curve and $\eta$ is a retraction of $X$ onto a core circle, then $B$ is an aposyndetic, homogeneous continuum that is not locally connected-in fact, $B$ is homeomorphic to the examples described in [8 and 4] if $G$ is the dyadic integers. On the other hand, if $\eta$ is the quotient map of a continuous decomposition of a continuum $X$ into pseudo-arcs, then $h$ is the quotient map of a continuous decomposition of the continuum $B$ into pseudo-arcs.

This approach does not give the same information at the inverse limit approachits appeal lies in its elegance and quickness. If one wishes complete information on, for instance, the shape of $B$, it seems necessary to use the approach of [8], or,

Received by the editors November 22, 1982 . Presented to the Society at the meeting in Baton Rouge, Louisiana, on November 13, 1982.

1980 Mathematics Subject Classification. Primary 54F20, 54F50.

Key words and phrases. Continuum, homogeneous, aposyndetic, solenoid, universal curve, induced bundle.

${ }^{1}$ This research was partially supported by NSF grant number MCS-8191565. 
equivalently, to develop the induced bundle for each bonding map of the inverse sequence.

If $P=\left(p_{1}, p_{2}, \ldots\right)$ is a sequence of positive integers greater than one, the $P$-adic solenoid $S$ is the limit of the inverse sequence in which each factor space is $S^{1}$ and the bonding map $f_{n}^{n+1}: S^{1} \rightarrow S^{1}$ is defined by $f_{n}^{n+1}(z)=z^{p_{n}}$. If $f: S \rightarrow S^{1}$ is the projection of $S$ onto the first factor space $S^{1}$, then the kernel of $f$ is a group $G$ called the $P$-adic integers. The topological group $G$ is an abelian group whose underlying space is homeomorphic to the Cantor set. Since $G$ has a local cross-section in $S$, it follows from [12, p. 31] that $S$ is a fiber bundle over $S^{1}=S / G$ relative to the projection $f$ which assigns to each $b$ the coset $b G$. The fiber of the bundle is $G$ and the group is $G$ acting on the fiber by left translations.

2. Induced bundles. In this section, we collect a few obvious facts about induced bundles. We adopt the (second) definition of induced bundle in [12, p. 47] and the notation therein. In particular, if $\eta: X \rightarrow X^{\prime}$ is a map and $\mathscr{B}^{\prime}=\left(B^{\prime}, p^{\prime}, X^{\prime}, Y, G\right)$ is a bundle with base space $X^{\prime}$, then the bundle space $B$ of the induced bundle $\eta^{-1} \mathscr{R}^{\prime}$ is the subspace of $X \times B^{\prime}$ consisting of those pairs $\left(x, b^{\prime}\right)$ such that $\eta(x)=p^{\prime}\left(b^{\prime}\right)$. The bundle map $p: B \rightarrow X$ is the projection onto the first coordinate, and the induced map $h: B \rightarrow B^{\prime}$ is the projection into the second coordinate (note that if $\eta$ is surjective, so is $h$ ). Thus the following diagram is commutative:

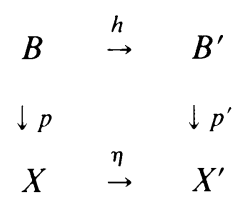

If $p^{\prime}\left(b^{\prime}\right)=x^{\prime}$, then $h^{-1}\left(b^{\prime}\right)=\left\{\left(x, b^{\prime}\right): \eta(x)=x^{\prime}\right\}$. Hence $h^{-1}\left(b^{\prime}\right)$ is homeomorphic to $\eta^{-1}\left(x^{\prime}\right)$, which implies the following useful fact.

PROPOSITION 1. If each point inverse of $\eta$ has some topological property, then so does each point inverse of $h$.

For example, if $\eta$ is monotone, acyclic, or cell-like, so is $h$, and if the point inverses of $\eta$ are pseudo-arcs, so are those of $h$.

Proposition 2. If $\eta$ is an open map, so is $h$.

Proof. Without loss of generality, an open set in $B$ is of the form $\phi_{j}\left(V_{j} \times W\right)$, when $V_{j}$ is an open subset of $X$ and $W$ is an open subset of $Y$. Then

$$
h\left(\phi_{j}\left(V_{j} \times W\right)\right)=h\left(V_{j}, \phi_{j}^{\prime}\left(\eta\left(V_{j}\right), W\right)\right)=\phi_{j}^{\prime}\left(\eta\left(V_{j}\right), W\right) \cong \eta\left(V_{j}\right) \times W .
$$

Hence $h$ is an open map.

3. Bundles induced from solenoid bundles. Throughout this section, $\eta: X \rightarrow S^{1}$ is a map of continuum $X$ into $S^{1}$, and $S$ is the $P$-adic solenoid bundle $\left(S, f, S^{1}, G\right)$. The bundle space $B$ of the induced bundle $\eta^{-1} S$ is always a nonlocally connected compactum of the same dimension as $X$.

Often $B$ is not a continuum. For instance, if $\eta$ is inessential, then $\eta^{-1} \delta$ is equivalent to the product bundle. If $X=S$ and $\eta=f$, then $\eta^{-1} \delta$ is the square of the 
bundle $\mathcal{S}$ and is equivalent to a product bundle; so even if $\eta$ is essential, $B$ may not be a continuum.

The following two theorems give conditions sufficient to prove that in the examples of interest in this paper, $B$ is a continuum.

THEOREM 3. If there exists a simple closed curve $C$ in the arcwise-connected continuum $X$ such that the restriction of $\eta$ to $C$ is a homeomorphism, then $B$ is a continuum.

Proof. The bundle space of $(\eta \mid C)^{-1}$ S is a solenoid $S$. For each $b$ in $B$, there exists an $\operatorname{arc}$ in $X$ from $p(b)$ to a point of $C$; this lifts to an arc from $b$ to a point of $S$. Hence $B$ is connected.

THEOREM 4. If $\eta: X \rightarrow S^{1}$ is monotone, then $B$ is a continuum.

Proof. It $\eta$ is monotone, so is $h$, by Proposition 1 . It is well known that the domain of a monotone map onto a continuum is itself a continuum. Hence $B$ is connected.

A continuum is colocally (arcwise-) connected if each of its points has arbitrarily small neighborhoods with (arcwise-) connected complements.

A continuum $X$ is aposyndetic at the point $p$ in $X$ if given any other point $q$ of $X$, there exists a subcontinuum of $X$ missing $q$ and containing $p$ in its interior. A continuum is aposyndetic if it is aposyndetic at each of its points. Note that colocally connected continua are aposyndetic.

The next two theorems give conditions sufficient for the continuum $B$ to be aposyndetic.

THEOREM 5. Let $X$ be a colocally arcwise-connected continuum. Suppose, for some two disjoint, simple closed curves in $X$, the restriction of $\eta$ to each is a homeomorphism. Then $B$ is a colocally connected (hence aposyndetic) continuum.

Proof. Theorem 3 implies that $B$ is a continuum. A point $b$ in $B$ has arbitrarily small neighborhoods of the form $\phi(V \times W)$, where $\phi$ is a coordinate transformation, $V$ is an open subset of a coordinate neighborhood, and $W$ is an open subset of $G$. We may assume $X-V$ is arcwise-connected and contains one of the simple closed curves; call this simple closed curve $C$. The bundle space of $(\eta \mid C)^{-1} \mathcal{S}$, being a solenoid $S$, is connected. If $b_{1}$ is a point of $B$ not in $p^{-1}(V)$, then there is an arc in $X$ from $p\left(b_{1}\right)$ to $C$, which lifts to an $\operatorname{arc}$ in $B$ from $b_{1}$ to $S$.

If $b_{2}=\phi(x, y), y \notin W$, let $K$ be the closure of the component of $\phi(V \times\{y\})$ containing $b_{2}$. Since the continuum $K$ contains a point of $B-p^{-1}(V)$, the result of the previous paragraph implies that there exists a continuum from $b_{2}$ to $S$ missing $\phi(V \times W)$. It follows that $B-\phi(V \times W)$ is connected.

THEOREM 6. Suppose each point of $X$ has arbitrarily small neighborhoods with connected boundary. If $B$ is a continuum, then $B$ is colocally connected (hence aposyndetic).

Proof. A point $B$ of $B$ has arbitrarily small neighborhoods $U^{\prime}$ of the form $\phi\left(V^{\prime} \times W\right)$, where $\phi$ is a coordinate transformation, $V^{\prime}$ is an open subset of a 
coordinate neighborhood, and $W$ is an open subset of $G$. Let $V$ be a neighborhood of $p(b)$ with boundary $Z$ such that $\bar{V} \subset V^{\prime}$ and $Z$ is connected. Let $U=\phi(V \times W)$. We shall show that $B-U$ is connected.

If $B-U=C \cup D$, where $C$ and $D$ are two disjoint, closed subsets of $B$, then no component of $p^{-1}(Z)$ intersects both $C$ and $D$. Define $C^{\prime}$ to be the union of all the components $K$ of $U$ satisfying $\bar{K} \cap C \neq \varnothing$. Define $D^{\prime}$ similarly. The $C \cup C^{\prime}$ and $D \cup D^{\prime}$ are two disjoint, closed sets whose union is $B$. Since $B$ is connected, either $C$ or $D$ is empty. Hence $B-U$ is connected. This completes the proof.

The space $X$ is stably homogeneous over the open subset $U$ if for each pair of points $x$ and $y$ of $U$, there exists a homeomorphism $h:(X, x) \rightarrow(X, y)$ such that $h$ is the identity on the complement of $U$.

The bundle 9 is stably homogeneous if there exists an open cover of the base space $X$ refining the collection of coordinate neighborhoods of $X$ such that $X$ is stably homogeneous over each element of this cover.

THEOREM 7. If $\left(B^{\prime}, X^{\prime}, p^{\prime}, G^{\prime}\right)$ is a stably homogeneous principal bundle with $X^{\prime} a$ continuum and $G^{\prime}$ abelian, then the bundle space $B^{\prime}$ is homogeneous.

Proof. Let $b_{1}$ and $b_{2}$ be two points of $B^{\prime}$ in the same fiber. Since $G^{\prime}$ is abelian, right translations of $B^{\prime}$ by elements of $G^{\prime}$ are bundle mappings that map each fiber onto itself $\left[12\right.$, p. 40], so there exists a homeomorphism of $\left(B^{\prime}, b_{1}\right)$ onto $\left(B^{\prime}, b_{2}\right)$.

Let $V$ be a coordinate neighborhood of $X^{\prime}$ containing $x$. Let $U$ be an open subset of $V$ containing $x$ such that $X^{\prime}$ is stably homogeneous over $U$. For any point $z$ in $U$, there exists a homeomorphism $k:\left(X^{\prime}, x\right) \rightarrow\left(X^{\prime}, z\right)$ that is the identity on the complement of $U$. Define $\tilde{k}: B^{\prime} \rightarrow B^{\prime}$ by letting $\tilde{k}$ be the identity map on the complement of $\left(p^{\prime}\right)^{-1}(U)$, and the product of $k$ and the identity map of the Cantor set on $\left(p^{\prime}\right)^{-1}(U)$.

Finite compositions of these two types of homeomorphisms provide enough homeomorphisms to show $X^{\prime}$ is homogeneous. This completes the proof.

The space $X$ is strongly locally homogeneous if each point of $X$ has arbitrarily small open neighborhoods over which $X$ is stably homogeneous.

COROLlaRY 8. If $X$ is strongly locally homogeneous, then the bundle space $B$ is homogeneous.

The last theorem of this section points out some topological properties $B$ must possess if it is a continuum.

THEOREM 9. If $B$ is a continuum, then $B$ is neither arcwise-connected nor movable, and $H^{1}(B ; Z)$ contains an element that admits infinite division.

Proof. Since $B$ is a continuum, $\eta$ is essential, for otherwise $\eta^{-1} \delta$ would be a product bundle. Hence $\eta$ is surjective, and so is $h$. Since $h$ maps the continuum $B$ onto the indecomposable continuum $S, B$ is not arcwise-connected. By [9], $H^{1}(B ; Z)$ contains an element that admits infinite division, and by [9, Corollary 4$], B$ is not movable.

4. Examples. The universal plane curve can be constructed by taking a square with boundary $A$, deleting the interior of the middle-ninth of that square, then deleting 
the interiors of the middle-ninths of each of the eight squares remaining, and continuing to delete middle-ninths of remaining squares. Each point of the universal plane curve has arbitrarily small neighborhoods with connected boundary.

EXAmple 1. Let $X$ be the universal plane curve, and let $\eta: X \rightarrow S^{1}$ be the composition of a retraction of $X$ onto $A$ and a homeomorphism of $A$ onto $S^{1}$. Then the bundle space $B$ is an aposyndetic, nonlocally connected, one-dimensional continuum.

EXAMPLE 2. The universal curve $M$ can be described as the set of all points of the unit cube that project, in each of the $x, y$, and $z$ directions, onto universal plane curves constructed (as above) on the faces of this cube. Each point of $M$ has arbitrarily small neighborhoods with connected boundary. R. D. Anderson [1] has shown that $M$ is strongly locally homogeneous. Let $X^{\prime}=M$, and let $\eta^{\prime}: M \rightarrow S^{1}$ be the composition of the map $\eta$ of Example 1 and the projection of $M$ into $R^{2}$. Then the bundle space $B^{\prime}$ of the induced bundle $\eta^{\prime-1} \delta$ is an aposyndetic, homogeneous, nonlocally connected, one-dimensional continuum.

EXAMPLE 3. Let $X$ be the circle of pseudo-arcs [2] and let $\eta: X \rightarrow S^{1}$ be an open map onto $S^{1}$ such that point inverses are pseudo-arcs. Then the bundle space $B$ is a solenoid of pseudo-arcs [10]; in fact, the map $h: B \rightarrow S$ defines a continuous decomposition of $B$ into pseudo-arcs such that the quotient space is a solenoid. The continuum $B$ is not aposyndetic, but it is homogeneous, since $\eta^{-1} \mathcal{S}$ is a stably homogeneous bundle. Note that $X$ is not a strongly locally homogeneous space. The fact that $B$ is circularly chainable does not follow from results of this paper, but rather from [8].

EXAMPLE 4. Suppose $X$ is a closed $n$-manifold of dimension greater than one, and $r: X \rightarrow A$ is a retraction of $X$ onto a simple closed curve $A$ in $X$. Let $\eta: X \rightarrow S^{1}$ be the composition of the map $r$ and a homeomorphism of $A$ onto $S^{1}$. Then $B$ is an aposyndetic, homogeneous, nonlocally connected continuum of dimension $n$.

EXAMPLE 5. Suppose there exists a continuum $X$ and an open map $\eta: X \rightarrow M$ of $X$ onto the universal curve $M$ such that point inverses are pseudo-arcs. Consider the induced bundle $\eta^{-1}\left(\eta^{\prime-1} \delta\right)$, where $\eta^{\prime-1} \mathcal{S}$ is the bundle of Example 2. The bundle space $B$ of $\left.\eta^{-1}\left(\eta^{\prime-1} \delta\right)\right)$ is a one-dimensional continuum that admits an open map $h$ : $B \rightarrow B^{\prime}$ onto the bundle space $B^{\prime}$ of Example 2 such that point inverses are pseudo-arcs. Moreover, if $\eta^{-1}\left(\eta^{\prime-1} \delta\right)$ is a stably homogeneous bundle, then $B$ is homogeneous.

5. Case continua. Let us call a continuum a Case continuum if it is homeomorphic to one of the continua constructed in Example 2 of the previous section. J. H. Case [4] constructed the first Case continuum via inverse limits. A Case continuum is homogeneous, aposyndetic, one-dimensional, and not locally connected. In this section we show there are uncountably many topologically distinct Case continua.

One can compute the Čech cohomology of a Case continuum directly from an inverse limit representation of the continuum; the following proof, however, seems more interesting.

THEOREM 10. Let $B$ be a Case continuum that retracts onto the solenoid $S$. Then $H^{1}(B ; Z) \cong H^{1}(S ; Z) \oplus \sum_{i=1}^{\infty} Z$. 
Proof. A retraction of $B$ onto $S$ implies

$$
H^{1}(B ; Z) \cong H^{1}(S ; Z) \oplus H^{1}(B, S ; Z) .
$$

The strong excision property of Alexander-Čech cohomology [11, p. 318] implies $H^{1}(B, S ; Z) \cong H^{1}(B / S ; Z)$, where $B / S$ is the quotient space obtained by collapsing $S$ to a point. The continuum $B / S$ is arcwise-connected, by the proof of Theorem 3 ; hence it is pointed one-movable [6]. Since $B / S$ is one-dimensional, it has the shape of a plane continuum [13] and hence of a (obviously infinite) bouquet of circles [3]. Since Alexander-Čech cohomology is a shape invariant, $H^{1}(B / S ; Z) \cong$ $\sum_{i=1}^{\infty} Z$. The conclusion of the theorem follows.

If $P_{\alpha}$ and $P_{\beta}$ are sequences of primes, define $P_{\alpha} \sim P_{\beta}$ if a finite number of terms can be deleted from each sequence so that every prime occurs the same number of times in the deleted sequences. Let $S_{\alpha}$ and $S_{\beta}$ be the solenoids associated with $P_{\alpha}$ and $P_{\beta}$, respectively. The following theorem is well known (see, for instance, [7, p. 198]).

THEOREM 11. The following are equivalent.

(1) $P_{\alpha} \sim P_{\beta}$.

(2) $S_{\alpha}$ is homeomorphic to $S_{\beta}$.

(3) $H^{\mathrm{l}}\left(S_{\alpha} ; Z\right) \cong H^{1}\left(S_{\beta} ; Z\right)$.

In fact, if $P_{\alpha}=\left(p_{1}, p_{2}, \ldots\right)$, then $H^{1}\left(S_{\alpha} ; Z\right)$ is isomorphic to the group of all rationals of the form $m /\left(p_{1} p_{2} \ldots p_{n}\right)$, where $m$ is an integer and $n$ is a positive integer.

THEOREM 12. There exist c topologically distinct Case continua.

Proof. Let $\left\{P_{\alpha}\right\}$ be a collection of $c$ sequences of primes with the property that any prime occurs at most once in any sequence and any two sequences have at most a finite number of primes in common. Associated with each $P_{\alpha}$ is a solenoid $S_{\alpha}$, a Case continuum $B_{\alpha}$, and a retraction of $B_{\alpha}$ onto $S_{\alpha}$. It follows from the previous theorem that if $\alpha \neq \beta$, then $H^{1}\left(S_{\alpha} ; Z\right)$ is not isomorphic to $H^{1}\left(S_{\beta} ; Z\right)$. Hence, from elementary considerations, $H^{1}\left(S_{\alpha} ; Z\right) \oplus \sum_{i=1}^{\infty} Z$ is not isomorphic to $H^{1}\left(S_{\beta} ; Z\right) \oplus$ $\sum_{i=1}^{\infty} Z$. Hence $B_{\alpha}$ is not homeomorphic to $B_{\beta}$, by Theorem 10 .

\section{REFERENCES}

1. R. D. Anderson, One-dimensional continuous curves and a homogeneity theorem, Ann. of Math. (2) 68 (1958), 1-16.

2. R. H. Bing and F. B. Jones, Another homogeneous plane continuum, Trans. Amer. Math. Soc. 90 (1959), 171-192.

3. K. Borsuk, Concerning homotopy properties of compacta, Fund. Math. 62 (1968), 223-254.

4. J. H. Case, Another 1-dimensional homogeneous continuum which contains an arc, Pacific J. Math. 11 (1961), 455-469.

5. F. B. Jones, Aposyndesis revisited, Proc. Univ. of Oklahoma Topology Conference, Norman, Oklahoma, 1972, pp. 64-78.

6. J. Krasinkiewicz and P. Minc, Generalized paths and pointed 1-movability, Fund. Math. 104 (1979), 141-153.

7. M. C. McCord, Inverse limit sequences with covering maps, Trans. Amer. Math. Soc. 114 (1965), 197-209. 
8. J. T. Rogers, Jr., An aposyndetic homogeneous curve that is not locally connected, Houston J. Math. (to appear).

9. A cohomological characterization of preimages of nonplanar circle-like continua, Proc. Amer. Math. Soc. 49 (1975), 232-236.

10. __ Solenoids of pseudo-arcs, Houston J. Math. 3 (1977), 531-537.

11. E. Spanier, Algebraic topology, McGraw-Hill, New York, 1966.

12. M. Steenrod, The topology of fibre bundles, Princeton Univ. Press, Princeton, N. J., 1951

13. A. Trybulec, On shapes of movable spaces, Bull. Acad. Polon. Sci. 21 (1973), 727-733.

Department of Mathematics, Tulane University, New Orleans, Louisiana 70118 